\title{
A 2D Numerical Model for Simulating the Physics of Fault Systems
}

\author{
Peter Mora ${ }^{1,2}$ and Dion Weatherley ${ }^{1}$ \\ ${ }^{1}$ Queensland University Advanced Centre for Earthquake Studies (QUAKES) \\ The University of Queensland, Brisbane, QLD 4072, Australia \\ ${ }^{2}$ The Australian Computational Earth Systems Simulator \\ Major National Research Facility (ACcESS MNRF) \\ The University of Queensland, Brisbane, QLD 4072, Australia
}

\begin{abstract}
Simulations provide a powerful means to help gain the understanding of crustal fault system physics required to progress towards the goal of earthquake forecasting. Cellular Automata are efficient enough to probe system dynamics but their simplifications render interpretations questionable. In contrast, sophisticated elasto-dynamic models yield more convincing results but are too computationally demanding to explore phase space. To help bridge this gap, we develop a simple 2D elastodynamic model of parallel fault systems. The model is discretised onto a triangular lattice and faults are specified as split nodes along horizontal rows in the lattice. A simple numerical approach is presented for calculating the forces at medium and split nodes such that general nonlinear frictional constitutive relations can be modeled along faults. Single and multi-fault simulation examples are presented using a nonlinear frictional relation that is slip and slip-rate dependent in order to illustrate the model.
\end{abstract}

\section{Introduction}

Numerical shear experiments of granular regions have exhibited accelerating energy release in the lead-up to large events[3] and a growth in correlation lengths in the stress field[4]. While these results suggest a Critical Point-like mechanism in elasto-dynamic systems and the possibility of earthquake forecasting, they do not prove that such a mechanism occurs in the crust. Cellular Automaton (CA) models exhibit accelerating energy release prior to large events or unpredictable behaviour in which large events may occur at any time, depending on tuning parameters such as the dissipation factor and stress transfer ratio[6]. The mean stress plots of the granular simulations are most similar to the CA mean stress plots near the boundary of the predictable and unpredictable regimes suggesting that elasto-dynamic systems may be close to the borderline between the predictable and unpredictable. To progress in resolving the question of whether more realistic fault system models exhibit predictable behaviour and to determine whether they also have an unpredictable and predictable regime depending on tuning parameters as seen in CA simulations, we develop a 2D elasto-dynamic model of parallel interacting faults. 
Preliminary work using the model[5] to simulate the dynamics of multiple parallel interacting faults have been performed and indicate, through calculations of the so called "inverse metric" (see [1]), that the system is non-ergodic. This has major implications to the analysis of crustal fault systems within a statistical physics framework. This work has also shown that such fault models exhibit so called "glassy" behaviour which implies that mean field theoretical analysis such as [1] require revision to introduce a memory kernel.

The elasto-dynamic parallel interacting fault model developed here may help provide a crucial link between CA maps of phase space and the behaviour of more realistic elasto-dynamic interacting fault system models, and thus, a means to improve understanding of the dynamics and predictability of real fault systems.

\section{$2 \quad$ Numerical Model}

The numerical model consists of a 2D triangular lattice of masses each of which is connected to its six nearest neighbours by a linear spring. This discretisation yields isotropic elasticity with compressional and shear wave speeds related by $V_{s}=V_{p} / \sqrt{3}[2]$ which is a typical value for rocks. This simple discretisation allows elasto-dynamics to be simulated relatively efficiently, albeit with the restriction of only one Poisson's ratio. Furthermore, horizontal faults can be easily specified in the model by splitting masses in half along a horizontal row, and simulating the frictional interaction of split masses with one another. Henceforth, we will refer to the masses as nodes of the discrete lattice. In the following, $\alpha=0, \ldots, 5$ is used to index the six lattice directions.

\section{Calculation of Forces}

The force on masses at medium nodes and at fault (split) nodes is calculated as

$$
\mathbf{F}_{i}=\left\{\begin{array}{lll}
\mathbf{F}_{i}^{e}+\mathbf{F}_{i}^{\nu}+\mathbf{F}_{i}^{T} & , \quad i \in M \\
\mathbf{F}_{i}^{f}+\mathbf{F}_{i}^{\nu}+\mathbf{F}_{i}^{T} & , \quad i \in F^{+} \text {or } i \in F^{-}
\end{array},\right.
$$

where subscript $i$ is used to denote mass (node) number $i, \mathbf{F}_{i}^{T}$ is a term representing "thermal noise" in the system, $\mathbf{F}_{i}^{e}$ is the elastic force due to springs connected to node $i$, and $\mathbf{F}_{i}^{f}$ is the sum of the elastic and frictional forces acting on the split nodes along faults.

\subsection{Elastic Forces}

The elastic forces are calculated by summing the elastic forces due to connected springs

$$
\mathbf{F}_{i}^{e}=\left\{\begin{array}{ll}
\sum_{\alpha=0}^{5} k\left(d^{\beta}-d_{0}\right) \mathbf{e}^{\beta} & , \quad i \in M \\
\left(\mathbf{F}_{i}^{e}\right)^{+} & , \quad i \in F^{+} \\
\left(\mathbf{F}_{i}^{e}\right)^{-} & , \quad i \in F^{-}
\end{array},\right.
$$


where $\beta=\bmod (\alpha, 6)$ is the lattice direction number, $k$ is the spring constant, $d^{\beta}$ is the distance to the mass linked in direction $\beta, d_{0}$ is the equilibrium separation or lattice spacing, $M$ denotes the set of medium nodes, $F^{+}$denotes the set of upper fault surface split nodes, and $F^{-}$denotes the set of lower fault surface split nodes. In the above equation, $\left(\mathbf{F}_{i}^{e}\right)^{+}$is the force on the upper split nodes due to linked nodes above the fault, and $\left(\mathbf{F}_{i}^{e}\right)^{-}$is the force of lower split nodes due to linked nodes below the fault, namely

$$
\begin{gathered}
\left(\mathbf{F}_{i}^{e}\right)^{+}=\sum_{\alpha=0}^{3} k^{\beta}\left(d^{\beta}-d_{0}\right) \mathbf{e}^{\beta} \quad i \in F^{+} \\
\left(\mathbf{F}_{i}^{e}\right)^{-}=\sum_{\alpha=3}^{6} k^{\beta}\left(d^{\beta}-d_{0}\right) \mathbf{e}^{\beta} \quad i \in F^{-},
\end{gathered}
$$

where

$$
k^{\alpha}=\left\{\begin{array}{ll}
k^{\alpha}=k & , \quad \alpha=1,2,4 \text { or } 5 \\
k^{\alpha}=k / 2 & , \quad \alpha=0,3
\end{array},\right.
$$

specifies spring constants connected to split nodes (i.e. spring constants in the medium are $k$ whereas the horizontal springs along the fault are split in two so $k^{\alpha}=k / 2$ for $\left.\alpha=0,3\right)$. As such, the total elastic force of split node pairs moving as a single mass $m$ in unison (i.e. when split nodes are in static frictional contact) is the sum of the elastic forces on the split node pair $\mathbf{F}_{i}^{e}=\left(\mathbf{F}_{i}^{e}\right)^{+}+\left(\mathbf{F}_{i}^{e}\right)^{-}$ and yields the same expression for force as for the medium nodes specified by Equation (2).

\subsection{Viscous Forces}

In order to damp energy in the closed elastic system, an artificial viscosity is introduced that attenuates elastic waves as $\exp (-\gamma t)$ where $\gamma$ is the frequency independent attenuation coefficient which is related to the viscosity coefficient by $\gamma=\nu / 2 m$ (e.g. see [2]). The viscous force is given by

$$
\mathbf{F}_{i}^{\nu}=-\nu\left(v_{i}-v_{0}\right),
$$

where $\nu$ is the viscosity coefficient, $v_{i}$ is the velocity of node $i$, and $v_{0}$ is a specified reference velocity (e.g. in constant strain rate shear experiments, we set $v_{0}$ to the velocity of a homogeneous elastic system undergoing shear). To have an attenuation coefficient $\gamma$ that is uniform in space, the viscosity $\nu$ must be set to $\nu=2 m \gamma$. Hence, for a homogeneous medium, the viscosity coefficients at split nodes are half as large as at medium nodes.

\subsection{Thermal Noise}

Recent research $[1,4-6]$ suggests that fault system models may be understood using concepts developed in statistical physics and that their dynamics may have 
similarities to classical Critical-Point systems. A key parameter in such systems is the temperature. As such, we introduce a thermal noise term to provide a means to study the statistical physics of the system. This is achieved by adding a random forcing term at each time step

$$
\mathbf{F}_{i}^{T}=\left\{\begin{array}{ll}
N_{i} & , \quad i \in M \\
N_{i} / 2 & , \quad i \in F^{+} \text {or } i \in F^{-}
\end{array},\right.
$$

where the magnitude of $N_{i}$ relates to the effective temperature of the system. The factor of a half for the second case is because the noise - which is assumed to have uniform statistics in space - is shared equally by each of the split nodes on faults. This term models random time dependent fluctuations in stress within the earth due to seismic background noise (distant earthquakes, earth tides, human noise, wind and ocean noise, etc).

\subsection{Vertical Component of Elastic Forces on Split Nodes}

The term $\mathbf{F}^{f}$ in Equation (1) represents the force on the split nodes due to medium elasticity and the friction. When split nodes are in contact, half of the vertical elastic force due to linked springs is applied to the split node itself and half is applied to the touching split node

$$
F_{y}^{f}=\left[\left(F_{y}^{e}\right)^{+}+\left(F_{y}^{e}\right)^{-}\right] / 2 .
$$

Hence, considering the mass of split nodes is $m / 2$, split nodes in contact accelerate in unison with vertical acceleration $\left[\left(F_{y}^{e}\right)^{+}+\left(F_{y}^{e}\right)\right] / m$ (i.e. the same vertical acceleration as a medium node at the split node location linked to the six neighboring nodes). When split nodes move out of contact (i.e. when $\left(F_{y}^{e}\right)^{+}-\left(F_{y}^{e}\right)^{-}>0$, there is no interaction between the split nodes so the vertical force on each split node is the elastic force due to its linked springs. Summarising the above, the vertical elastic force on split nodes is given by

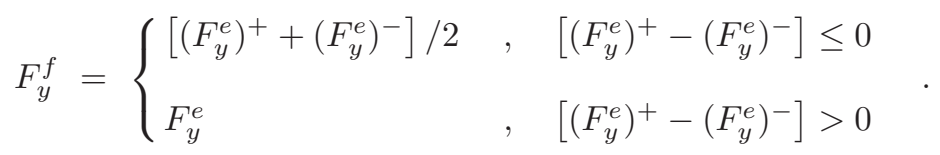

\subsection{Horizontal Component of Frictional and Elastic Forces on Split Nodes}

The horizontal force on a split node is the sum of the horizontal elastic force due to linked springs and a frictional force $f$

$$
F_{x}^{f}=F_{x}^{e}+f,
$$

where $F_{x}^{e}$ is the horizontal elastic force on the split node being considered given by Equation (3). In the case of a fault in static frictional contact, the friction is such that split nodes accelerate horizontally in unison so

$$
f=\left[\left(F_{x}^{e}\right)^{\prime}-F_{x}^{e}\right] / 2,
$$


where the ${ }^{\prime}$ is used to denote the other split node (ie. if we are calculating $f$ for the upper split node, then the ' signifies the lower split node and vice versa). Hence, for the case of static frictional contact, the split nodes both accelerate with a horizontal acceleration $\left[\left(F_{x}^{e}\right)^{+}+\left(F_{x}^{e}\right)^{-}\right] / m$ which is equal to the acceleration that would be calculated for a medium node of mass $m$ that replaces the split node pair (c.f. case for vertical forces). When the elastic force is sufficiently great to overcome static friction, the split nodes will begin to slip. Therefore, we can write

$$
|f|=\min \left(\left|\left[\left(F_{x}^{e}\right)^{\prime}-F_{x}^{e}\right] / 2\right|,|\tau|\right),
$$

where $\tau$ is a function that prescribes the frictional constitutive relation with $\tau^{+}=-\tau^{-}$(i.e. the friction is equal in magnitude and opposite in direction on the upper and lower fault surfaces). Hence, the friction can be written as

$$
f= \begin{cases}{\left[\left(F_{x}^{e}\right)^{\prime}-F_{x}^{e}\right] / 2} & , \quad\left|\left[\left(F_{x}^{e}\right)^{\prime}-F_{x}^{e}\right] / 2\right|<|\tau| \\ \tau & , \quad \text { otherwise }\end{cases}
$$

The sign of $\tau$ is such that it opposes slip between the nodes. As such, we can write

$$
\operatorname{sgn}(\tau)=\left\{\begin{array}{ll}
{\left[\left(F_{x}^{e}\right)^{\prime}-F_{x}^{e}\right] /\left|\left(F_{x}^{e}\right)^{\prime}-F_{x}^{e}\right|} & , \quad S(t-\Delta t)=\text { stick } \\
\left(V_{x}^{\prime}-V_{x}\right) /\left|V_{x}^{\prime}-V_{x}\right| & , \quad S(t-\Delta t)=\text { slip }
\end{array},\right.
$$

where $S(t-\Delta t)$ specifies the state of the split node at the previous time step $t-\Delta t$ and may be either stick or slip. The first case ensures the friction will oppose the new slip velocity of a split node pair that is beginning to slip whereas the second case opposes the slip velocity of an already slipping split node pair. Hence, in the upper case, the state changes from $S(t-\Delta t)=$ stick to $S(t)=$ slip whereas in the lower case, the state remains unchanged (i.e. $S(t)=S(t-\Delta t)=$ slip).

The magnitude of the friction is given by

$$
|\tau|=\mu\left[\left(F_{y}^{e}\right)^{-}-\left(F_{y}^{e}\right)^{+}\right] / 2,
$$

(i.e. Coulomb friction) where the friction coefficient $\mu$ may be a function of dynamic variables such as slip or slip-rate. In the following examples, we will use a friction that is slip and slip-rate dependent as follows

$$
\mu(t)=\left\{\begin{array}{ll}
\mu_{d}+\left(\mu_{s}-\mu_{d}\right)\left(1-s(t) / D_{c}\right)^{p_{1}} & , \quad s<D_{c} \\
\mu_{d}+\left(\mu_{s}-\mu_{d}\right)\left(1-\dot{s}(t-\Delta t / 2) / V_{c}\right)^{p_{2}} & , \quad s \geq D_{c}
\end{array},\right.
$$

where $\mu_{s}$ and $\mu_{d}$ are respectively the static and dynamic friction coefficients, $p_{1}$ and $p_{2}$ are exponents that control the functional form of friction with slip and slip-rate, $s$ is the amount of slip during a rupture event, $\dot{s}$ is the slip-rate, $D_{c}$ is the "critical slip weakening distance" over which friction weakens to the dynamic value, and $V_{c}$ is the slip-rate when slip reaches $D_{c}$. The slip-rate is 
calculated using centred finite differences from the positions of the nodes at the current and past time steps which is the reason why $\dot{s}$ is at time $(t-\Delta t / 2)$. This frictional relation is slip weakening until the slip equals $D_{c}$ and then remains at the dynamic value until the slip-rate drops below the value it attained when slip first exceeded $D_{c}$. As the slip-rate drops further, the fault re-strengthens as a function of velocity and reaches the static friction once the slip-rate drops to zero. The second term allows the fault to heal after passage of a rupture front and yields slip-pulse behaviour of simulated earthquake ruptures consistent with observations, rather than less realistic crack-like ruptures which result from simple slip weakening relationships. Exponent $p_{1}$ controls the sharpness of the leading edge of the pulse and $p_{2}$ controls the sharpness of the trailing edge of the pulse. In the following examples, we will set $p_{1}=2$ and $p_{2}=1$ which yields a relatively symmetrical slip pulse.

\section{Time Integration Scheme}

A second order finite difference scheme is used to extrapolate the positions in time as follows

$$
\left(u_{i}\right)_{\ell}(t+\Delta t)=2\left(u_{i}\right)_{\ell}(t)-\left(u_{i}\right)_{\ell}(t-\Delta t)+\Delta t^{2}\left(a_{i}\right)_{\ell}(t),
$$

where $\left(u_{i}\right)_{\ell}$ is the $\ell$-th component of the displacement at the $i$-th node and the acceleration $\mathbf{a}_{i}$ is calculated from the force given by Equation (1) as

$$
\mathbf{a}_{i}=\mathbf{F}_{i} / m_{i},
$$

where $m_{i}$ is the mass of the $i$-th node (e.g. for a homogeneous medium, $m$ would be constant except for split nodes which would have $\left.m_{i}=m / 2\right)$. Once the new displacements are calculated, we can evaluate the new slip-rate at time $(t+\Delta t / 2)$. When the slip-rate changes sign, i.e. when $\operatorname{sgn}(\dot{s}(t-\Delta t / 2))=-\operatorname{sgn}(\dot{s}(t+\Delta t / 2)$, it is assumed that slip is stopping and the change in sign is caused by numerical overshoot due to the finiteness of the time step $\Delta t$. In this case, we set $S(t+\Delta t / 2)$ to stick.

\section{$5 \quad$ Numerical Examples}

In all examples, we set $\Delta t=0.4, d_{0}=\Delta x=1, k=1$ and $m=1$ and use lattice dimensions of $N_{x}=100$ and $N_{y}=101$. These parameters yield a P-wave speed of $V_{p} \approx 1$ (see [2]). In the single fault examples, a horizontal fault was centered in the model at $y=y_{50}$ where $y_{n}=n \Delta y$ denotes the $y$-ordinates of the $n$-th row of lattice nodes and row indices have the range $n=0, \ldots N_{y}-1$. In all cases, the vertical strain was fixed at $\varepsilon_{y y}=0.02$ and viscosity was set to $\nu=0.04$. Boundary conditions are circular in $x$ and rigid in $y$, and the thermal noise $N_{i}$ is set to 0 . 


\subsection{Waves Traveling through a Locked Fault}

A point source was excited below the fault with the aim of verifying that split nodes in static frictional contact behave identically as medium nodes. The snapshot shown in Figure 1 shows circular compressional and shear waves propagating through the locked fault. There are no artificial reflections, thus verifying the implementation for static frictional contact.

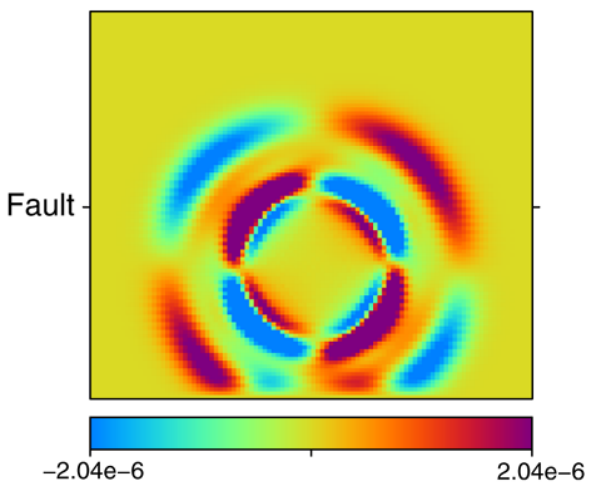

Fig. 1. Snapshot showing the vertical component of velocity at time $t=25$ due to a point source below the fault located at $\mathbf{x}=(50,34)$. The $y$-ordinate of the horizontal fault is indicated by tic marks on the frame. The source was excited by adding a Gaussian perturbation $K e^{-\kappa\left(t-t_{0}\right)}$ to the horizontal component of displacement. Source parameters were $K=0.0001, \kappa=0.05$ and $t_{0}=25$. The colour scale saturates when $\left|v_{y}\right|$ exceeds 0.3 times its maximum value.

\subsection{Rupture of a Homogeneous Fault}

A numerical shear experiment was conducted by driving the upper and lower rows of nodes, which represent rigid driving plates, in opposite directions at a constant rate of 0.0005 (i.e. approx 0.0005 times the $\mathrm{P}$-wave speed). The fault static friction was initialised to $\mu_{s}=0.8$ everywhere except at the middle node located at $x=50 \Delta x$, which was set was set to $\mu_{s}=0.75$. This provides a weak point or seed for the rupture to nucleate. The dynamic friction was set to $\mu_{d}=0.7$ everywhere and the critical slip weakening distance was set to $D_{c}=0.02$. Initially, there was no slip along the fault followed by quasi-static slip at the central weak point when the system became sufficiently stressed. As the stress builds up, a small region of slip grows quasi-statically around the weak node until a dynamic rupture is initiated which then propagates outwards at the compressional wave speed (Figures 2 and 3 ).

\subsection{Rupture of a Heterogeneous Fault}

A second shear experiment was conducted with the same parameters as the previous example except that both the static and dynamic friction were assigned values from a power law distribution, specifically $\mu\left(x_{n}\right)=f f t^{-1}\left\{k_{n}^{-p} N_{n}\right\}$ where exponent $p=0.3, N_{n}$ is white noise, and $x_{n}$ and $k_{n}$ respectively denote the discrete locations and wavenumbers. The range of fluctuations for the static friction was $\mu_{s} \in[0.7,0.8]$ and the range for the dynamic friction was [0.55, 0.65]. After several slip events, the stress becomes highly heterogeneous along the fault (Figure 4) and once this happens, ruptures typically propagate at approximately 


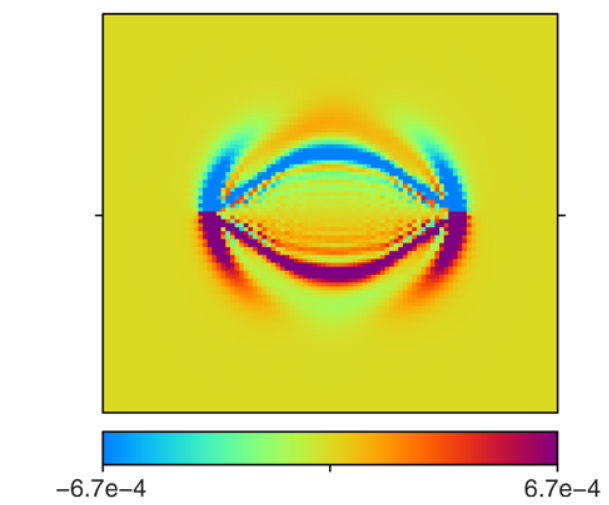

Fig. 2. Snapshot of the horizontal component of velocity showing a rupture propagating bi-directionally along the fault at the compressional wave speed. The $y$-ordinate of the horizontal fault is indicated by tic marks on the frame. The colour scale saturates when $\left|v_{x}\right|$ exceeds 0.1 times its maximum value thus allowing small amplitudes to be visualised.

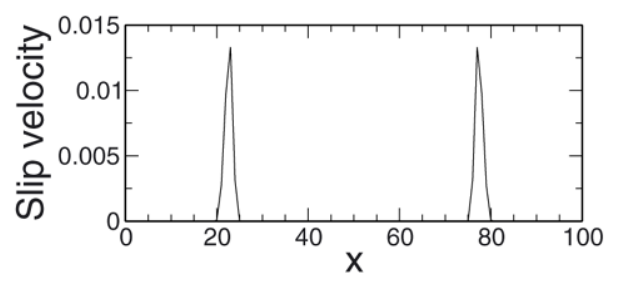

Fig. 3. Slip velocity on the fault defined as the horizontal velocity of upper split nodes subtracted from the horizontal velocity of lower split nodes.

the shear or Rayleigh wave speed (Figures 4 \& 5). Because of the heterogeneity, it is typical that the rupture front will propagate in one direction only and leave a complex wave train following the rupture front as shown in Figure 4 (right).

\subsection{Multi-fault Simulation}

As the motivation to develop the numerical model is to study the physics of interacting fault systems, we present a shear experiment with a number of parallel faults to illustrate this capability. The same parameters were used as in the single heterogeneous fault case except that 11 faults were initialised at $y=25+5 j \Delta y$ where $j=0, \ldots 11$ denotes the fault number. In this example, a lower shear rate was used than in previous examples. Namely, the speeds of the upper and lower rows were set to 0.0002 instead of 0.0005 . Figure 6 shows the shear stress as a function of time. The saw-tooth shapes are characteristic of stick-slip behaviour, with each drop being caused by a dynamic rupture event on a fault.

Figure 7 shows snapshots of the shear stress $\sigma_{x y}$ and horizontal component of velocity in the model at the 136050-th time step when a dynamic rupture event is occurring. The stress field in the fault region is complex and heterogeneous although coherent high stress bands can be seen running diagonally across the model. These are analogous to grain bridges which support stress in granular models. The numerical model allows the evolution of these complex stress patterns to be studied. One goal of such studies would be to determine whether there is a consistent growth in correlation lengths in the lead-up to large events in accord with the Critical Point Hypothesis for earthquakes and as seen in granular numerical models[4]. The horizontal component of velocity shows a rupture 

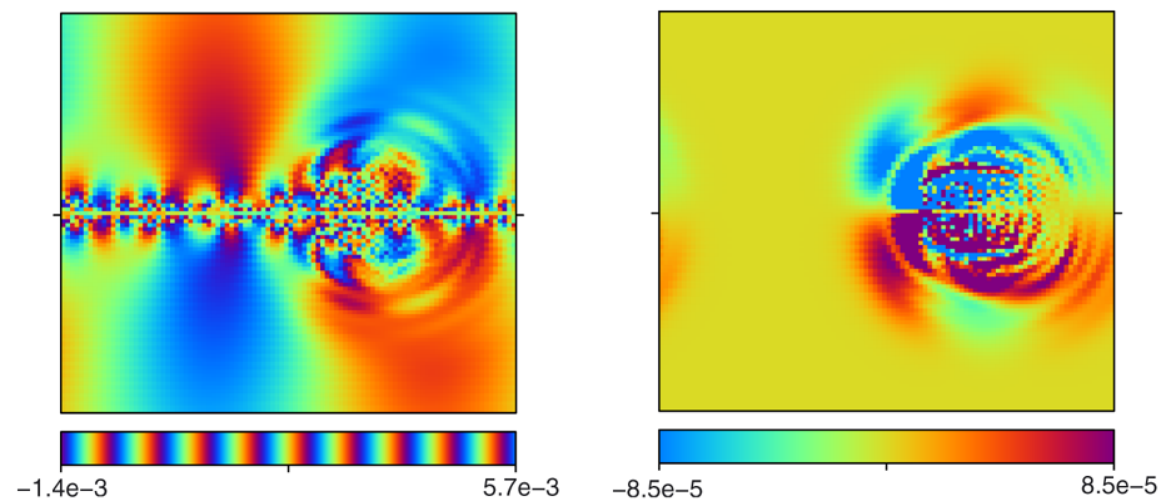

Fig. 4. Snapshots of $\sigma_{y y}$ (left) and horizontal component of velocity $v_{x}$ (right) showing a rupture propagating to the left at around the Rayleigh wave speed. The colour scale of $v_{x}$ saturates when $\left|v_{x}\right|$ exceeds 0.015 times its maximum value.

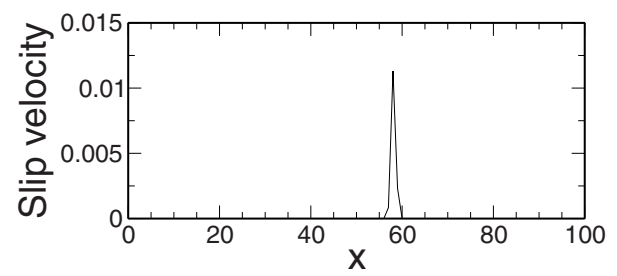

Fig. 5. Slip velocity on the fault at the same time as the snapshot shown in Figure 4.

propagating to the right along the 4-th fault from the bottom. In some cases, a rupture on one fault will trigger rupture on another fault. In most cases, ruptures propagate at around the Rayleigh wave speed although ruptures frequently accelerate to the $\mathrm{P}$-wave speed, probably as a consequence of the high driving rate. Both unidirectional and bidirectional ruptures were observed.

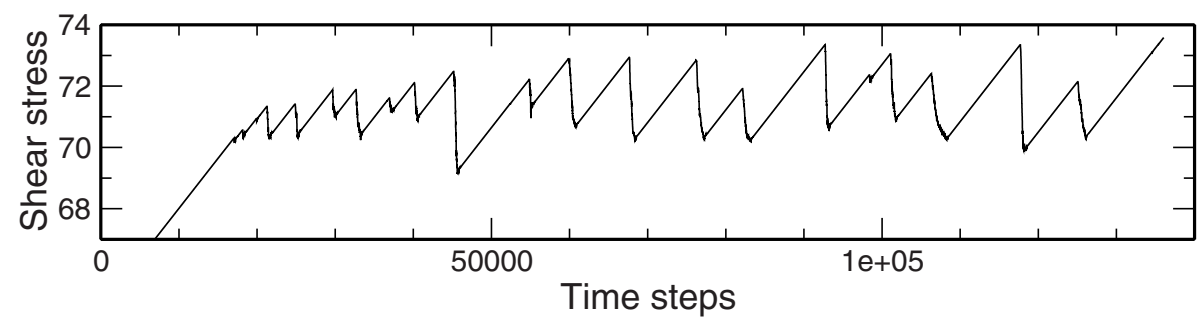

Fig. 6. Shear stress in the multi-fault model measured on the upper \& lower edges of the lattice. The plot shows characteristic saw-tooth shapes associated with stick-slip behaviour. The initial shear stress was non-zero to minimise the first loading time.

\section{Conclusions}

A simple and relatively efficient numerical model is presented that provides a means to simulate the physics of parallel fault systems, and hence, a means to 

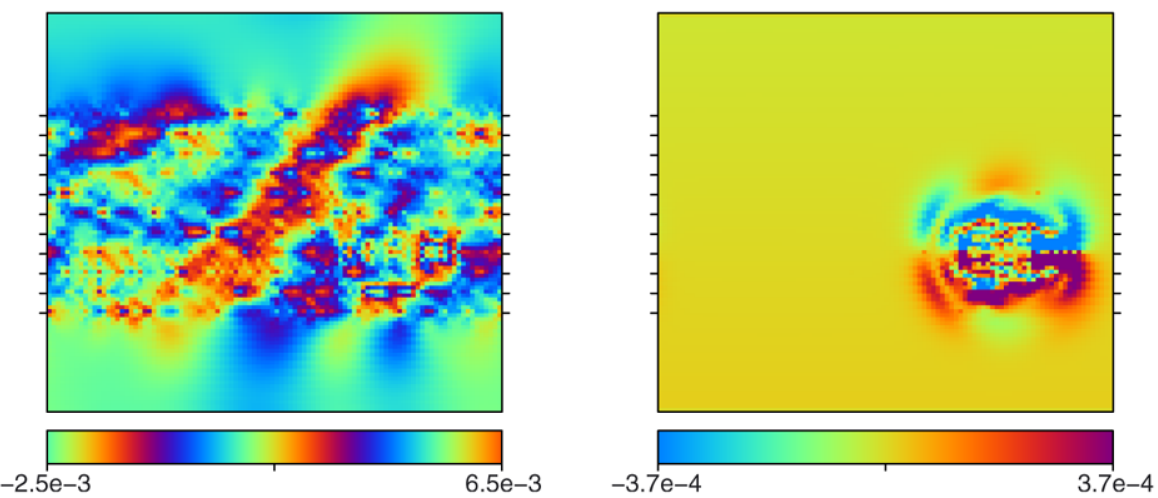

Fig. 7. Snapshots of shear stress (left) and the horizontal component of velocity (right). The colour scale of $v_{x}$ saturates when $\left|v_{x}\right|$ exceeds 0.05 times its maximum value. The $y$-ordinates of the 11 horizontal faults are indicated by tic marks on the frame.

study whether elasto-dynamic crustal fault models may exhibit a dynamics that enables earthquakes to be forecast.

\section{Acknowledgement.}

This work has been supported by The University of Queensland, the Australian Research Council, and the Australian Computational Earth Systems Simulator Major National Research Facility.

\section{References}

1. Klein W., and Anghel M., Ferguson C.D., Rundle J.B., and Martins, J.S.Sá (2000) Statistical analysis of a model for earthquake faults with long-range stress transfer, in: Geocomplexity and the Physics of Earthquakes (Geophysical Monograph series; no. 120), eds. Rundle, J.B. and Turcotte, D.L., and Klein, W., pp 43-71 (American Geophys. Union, Washington, DC).

2. Mora, P., and Place, D. (1994) Simulation of the frictional stick-slip instability, Pure Appl. Geophys., 143, 61-87.

3. Mora, P., Place, D., Abe, S. and Jaumé, S. (2000) Lattice solid simulation of the physics of earthquakes: the model, results and directions, in: GeoComplexity and the Physics of Earthquakes (Geophysical Monograph series; no. 120), eds. Rundle, J.B., Turcotte, D.L. \& Klein, W., pp 105-125 (American Geophys. Union, Washington, DC).

4. Mora, P., and Place, D. (2002) Stress correlation function evolution in lattice solid elasto-dynamic models of shear and fracture zones, and earthquake prediction, Pure Appl. Geophys, 159, 2413-2427.

5. Mora, P., Weatherley, D., and Klein, W. (2003) Simulation of parallel interacting faults and earthquake predictability, Proc. European Geophysical Society Annual Meeting.

6. Weatherley, D. and Mora, P. (2003) Accelerating precursory activity within a class of earthquake analog automata, Pure Appl. Geophysics, accepted. 